PROCEEDINGS OF THE

AMERICAN MATHEMATICAL SOCIETY

Volume 29, Number 3, August 1971

\title{
A WHITEHEAD TYPE THEOREM
}

\author{
H. B. HASLAM ${ }^{1}$
}

Abstract. Let $\mathfrak{F}$ denote the Serre class of finite abelian groups. We consider, for example, conditions under which a map which induces an $\mathfrak{F}$-epimorphism in homotopy also induces an $\mathfrak{F}$-epimorphism in homology.

1. Introduction. Let $f: X \rightarrow Y$ be a map, 5 a Serre class of abelian groups. Modulo some technical assumptions on the spaces $X$ and $Y$ or the class $\mathbb{E}$, the Whitehead theorem states that $f$ induces a $\mathbb{E}$ isomorphism in homotopy in each dimension if and only if it induces a 5 -isomorphism in homology in each dimension. We are concerned here with finding conditions under which the word "isomorphism" can be replaced by "epimorphism" or "monomorphism" for the class $\mathfrak{F}$ of finite abelian groups. For example, we show that if a map $g$ from an $H$-space $Y$ to a 1-connected finite CW-complex $X$ induces an $\mathfrak{F}$-epimorphism in homotopy, then it induces an $\mathfrak{F}$-epimorphism in homology and $X$ is an $H$-space mod $\mathfrak{F}$. As a special case we recover a result of [4]: If $X$ is a 1-connected finite CW-complex and a $G$ space $\bmod \mathfrak{F}$ (i.e. the evaluation map $\omega:\left(X^{x}, 1\right) \rightarrow(X, *)$ induces an $\mathfrak{F}$-epimorphism in homotopy), then $X$ is an $H$-space mod $\mathfrak{F}$. (The converse is also true.) Moreover, $\omega$ induces an $\mathfrak{F}$-epimorphism in homology. The proof given here is much simpler than that given in [4].

I wish to thank W. Browder for a conversation which motivated this note.

All spaces are assumed to have the based homotopy type of a CW-complex and all maps and homotopies are to preserve base points. We will frequently not distinguish between a map and its homotopy class. The symbol "h" will be used to denote Hurewicz homomorphisms. We assume that the reader is familiar with [1] and [2].

2. The result. Let $X$ be a 1 -connected finite CW-complex, $Y$ be a 1-connected space with $H_{m}(Y)$ finitely generated for all $m$ and let $f: X \rightarrow Y, g: Y \rightarrow X$ be maps.

Theorem 1. Suppose that $Y$ is an $H$-space.

Received by the editors August 18, 1970.

AMS 1969 subject classifications. Primary 5540.

Key words and phrases. $H$-space $(\bmod \mathfrak{F}), H^{\prime}$-space $(\bmod \mathfrak{F})$.

1 Supported by NSF Grant GP 19964.

Copyright @ 1971, American Mathematical Society 
(i) If $f_{*}: \pi_{m}(X) \rightarrow \pi_{m}(Y)$ is an $\mathfrak{F}$-monomorphism for all $m$, then so is $f_{*}: H_{m}(X) \rightarrow H_{m}(Y)$.

(ii) If $g_{*}: \pi_{m}(Y) \rightarrow \pi_{m}(X)$ is an $\mathfrak{F}$-epimorphism for all $m$, then so is $g_{*}: H_{m}(Y) \rightarrow H_{m}(X)$. Moreover, $X$, in each case, is an $H$-space mod $\mathfrak{F}$.

TheOREM 2. Suppose that $Y$ is an $H^{\prime}$-space.

(i) If $f_{*}: H_{m}(X) \rightarrow H_{m}(Y)$ is an $\mathfrak{F}$-monomorphism for all $m$, then so is $f_{*}: \pi_{m}(X) \rightarrow \pi_{m}(Y)$.

(ii) If $g_{*}: H_{m}(Y) \rightarrow H_{m}(X)$ is an $\mathfrak{F}$-epimorphism for all $m$, then so is $g_{*}: \pi_{m}(Y) \rightarrow \pi_{m}(X)$. Moreover, $X$, in each case, is an $H^{\prime}$-space mod $\mathfrak{F}$.

REMARK. The 1-connectedness assumption on $Y$ is needed only for Theorem 2 (ii) and neither assumption on $Y$ is needed for Theorem 1 (ii).

We will need the following result, the proof of which depends only on the universal coefficient theorem and the representability of cohomology.

LEMMA 1. Let $B$ be a space for which $H_{m}(B)$ is finitely generated and let $\beta \in \pi_{m}(B)$. Then $\mathfrak{h}(\beta) \neq 0$ if and only if there is a map $h: B \rightarrow K(\pi, m)$ (=an Eilenberg-Mac Lane space) such that $h \beta$ is not homotopic to a constant. (We may take $\pi=Z_{p^{r}}$ or $Z$ depending on whether $\mathfrak{h}(\beta)$ has finite or infinite order.)

Lemma 2. Let $a: A \rightarrow B$ be a map from an $H$-space $A$ to a finite $\mathrm{CW}$ complex $B$. If $a_{*}: \pi_{2 n}(A) \rightarrow \pi_{2 n}(B)$ is an $\mathfrak{F}$-epimorphism, then $\mathfrak{h}\left(\pi_{2 n}(B)\right)$ $\in \mathfrak{F}$.

Proof. It suffices to show that $\mathfrak{h}(\beta)$ has finite order for each $\beta \in \pi_{2 n}(B)$. In order to obtain a contradiction, assume that there is a $\beta \in \pi_{2 n}(B)$ such that $\mathfrak{h}(\beta)$ has infinite order. By Lemma 1 , there is a map $h: B \rightarrow K(Z, 2 n)$ such that $h \beta$ is not homotopic to a constant. Since $a_{*}: \pi_{2 n}(A) \rightarrow \pi_{2 n}(B)$ is an $\mathfrak{F}$-epimorphism there is an $\alpha \in \pi_{2 n}(A)$ such that $a_{*}(\alpha)=r \beta$ where $r$ is some nonzero integer. Now, if $\rho: \Omega \Sigma A$ $\rightarrow A$ is a retraction map ( $A$ is an $H$-space), then $h a \rho \circ \Omega \Sigma \alpha: \Omega \Sigma S^{2 n}$ $\rightarrow K(Z, 2 n)$ is a nontrivial map which factors through a finite complex. This is clearly impossible (consider the ring structure of $\left.H^{*}\left(\Omega \Sigma S^{2 n}\right)\right)$ and the lemma is proved.

Corollary 1. If $B$ is $(2 n-1)$-connected, then $\pi_{2 n}(B) \in \mathfrak{F}$.

Proof of Theorem 1. (i) Since the homotopy suspension homomorphism for an $H$-space is a monomorphism in all dimensions, it follows that the suspension homomorphism $i_{*}: \pi_{m}(X) \rightarrow \pi_{m}(\Omega \Sigma X)(i$ is the inclusion map) is an $\mathfrak{F}$-monomorphism for all $m$ and therefore that $X$ is an $H$-space $\bmod \mathfrak{F}$. Let $h: S \rightarrow X$ be a weak $\mathfrak{F}$-equivalence, 
where $S$ is a finite product of odd dimensional spheres. Since the Hurewicz homomorphism $\mathfrak{h}: \pi_{m}(Y) \rightarrow H_{m}(Y)$ is an $\mathfrak{F}$-monomorphism it follows from Lemma 1 that $f h$ induces an $\mathfrak{F}$-epimorphism in cohomology and hence an $\mathfrak{F}$-monomorphism in homology. Thus $f$ induces an $\mathfrak{F}$-monomorphism in homology.

(ii) We first show, by induction, that $\pi_{2 n}(X) \in \mathfrak{F}$ for all $n$. For $n=1$, this follows from Corollary 1 . Assume that $\pi_{2 n}(X) \in \mathfrak{F}$ for $2 n<N, N$ odd. Since $g_{*}: \pi_{m}(Y) \rightarrow \pi_{m}(X)$ is an $\mathfrak{F}$-epimorphism for all $m$, we can use the multiplication on $Y$ to obtain a map $h_{N}: S \rightarrow Y$ such that $g h_{N}$ induces an $\mathfrak{F}$-isomorphism in homotopy in dimensions $\leqq N$, where $S$ is a finite product of odd dimensional spheres $S^{n_{i}}$, $3 \leqq n_{i} \leqq N$. We can assume that $g h_{N}$ is an inclusion map. Then $\pi_{m}(X, S) \in \mathfrak{F}$ for all $m \leqq N$; by the Hurewicz theorem, $\mathfrak{h}: \pi_{N+1}(X, S)$ $\rightarrow H_{N+1}(X, S)$ is an $\mathfrak{F}$-isomorphism. Since $\pi_{N+1}(S) \in \mathfrak{F}$, it follows that $\mathfrak{h}: \pi_{N+1}(X) \rightarrow H_{N+1}(X)$ is an $\mathfrak{F}$-monomorphism and so, by Lemma 2, $\pi_{N+1}(X) \in \mathfrak{F}$. Thus $\pi_{2 n}(X) \in \mathfrak{F}$ for all $n$. It is now a simple matter to show that for $N \geqq \operatorname{dim} X, g h_{N}$ is a weak $\mathfrak{F}$-equivalence. Therefore $X$ is an $H$-space $\bmod \mathfrak{F}$ and $g_{*}: H_{m}(Y) \rightarrow H_{m}(X)$ is an $\mathfrak{F}$-epimorphism for all $m$.

Proof of Theorem 2. (i) Since $f_{*}: H_{m}(X) \rightarrow H_{m}(Y)$ is an $\mathfrak{F}$-monomorphism for all $m, f^{*}: H^{m}(Y) \rightarrow H^{m}(X)$ is an $\mathfrak{F}$-epimorphism for all $m$. Let $\left\{\beta_{i}\right\}$ be a basis for the free part of $H^{*}(X)$ and let $\left\{\gamma_{i}\right\} \subset H^{*}(Y)$ be chosen so that $f^{*}\left(\gamma_{i}\right)=t_{i} \beta_{i}$ for some nonzero integer $t_{i}$. Let $r>$ $\operatorname{dim} X$ be arbitrary, $\gamma_{i}^{\prime}=\gamma_{i} \mid Y^{r}$, where $Y^{r}$ is the $r$-skeleton of $Y$.

Since $\gamma_{i} \cup \gamma_{i}=0$ ( $Y$ is an $H^{\prime}$-space), $\gamma_{i}^{\prime} \cup \gamma_{i}^{\prime}=0$ and [3] there is a map $h_{i}: Y^{r} \rightarrow S^{n_{i}}, n_{i}=\operatorname{dim} \gamma_{i}$, which maps the fundamental class of $S^{n_{i}}$ to some nonzero multiple of $\boldsymbol{\gamma}_{i}^{\prime}$. Making use of the fact that $Y$ is an $H^{\prime}$-space we obtain a map $h: Y^{r} \rightarrow \bigvee S^{n_{i}}$ (as in [3]) such that $h f$ is a weak $\mathfrak{F}$-equivalence (by the cellular approximation theorem we can assume $f(X) \subset Y^{r}$ ). Therefore $X$ is an $H^{\prime}$-space mod $\mathfrak{F}$ and $f_{*}: \pi_{m}(X)$ $\rightarrow \pi_{m}(Y)$ is an $\mathfrak{F}$-monomorphism for $m<r$. Since $r$ was arbitrary the result follows.

(ii) Since the Hurewicz homomorphism for an $H^{\prime}$-space is an $\mathfrak{F}$ epimorphism in all dimensions, it follows that $\mathfrak{h}: \pi_{m}(X) \rightarrow H_{m}(X)$ is an $\mathfrak{F}$-epimorphism for all $m$ and hence that $X$ is an $H^{\prime}$-space $\bmod \mathfrak{F}$. Moreover, it is clear that there is a map $h: \bigvee S^{n_{i}} \rightarrow Y$ such that $f h$ is a weak $\mathfrak{F}$-equivalence and the result follows.

REMARK. In contrast to the Whitehead theorem, the converse of each assertion in Theorems 1 and 2 is false. Counterexamples are given as follows:

1(i). The inclusion map $S^{2 n} \rightarrow \Omega \Sigma S^{2 n}$.

1(ii). The quotient map $S^{n} \times S^{n} \rightarrow S^{n} \wedge S^{n}=S^{2 n}, n=3$ or 7 . 
2(i). The Whitehead product map $S^{m+n-1} \rightarrow S^{m} \bigvee S^{n}, m+n$ even, $m, n \geqq 2$.

2(ii). The inclusion map $S^{m} \bigvee S^{n} \rightarrow S^{m} \times S^{n}$.

\section{BIBLIOGRAPHY}

1. M. Arkowitz and C. R. Curjel, The Hurewicz homomorphism and finite homotopy invariants, Trans. Amer. Math. Soc. 110 (1964), 538-551. MR 28 \#1624.

2. - Zum Begriff des H-Raumes mod F, Arch. Math. 16 (1965), 186-190. MR 31 \#4031.

3. Israel Berstein, Homotopy mod (5 of spaces of category 2, Comment. Math. Helv. 35 (1961), 9-14. MR 23 \#A652.

4. H. B. Haslam, G-spaces mod $\mathfrak{F}$ and $H$-spaces mod $\mathfrak{F}$, Duke Math. J. (to appear).

Florida State University, Tallahassee, Florida 32306 